\title{
On Development of MySignals based prototype for application in health vitals monitoring
}

\author{
Sohail Saif $^{1}$ (D) $\cdot$ Ramesh Saha $^{2}$ (D) $\cdot$ Suparna Biswas $^{1}$ (D)
}

Accepted: 9 August 2021 / Published online: 18 August 2021

(C) The Author(s), under exclusive licence to Springer Science+Business Media, LLC, part of Springer Nature 2021

\begin{abstract}
India's health infrastructure is under pressure since the daily COVID-19 cases have crossed the milestone of 4 Lakhs cases per day which surpass the previous years' peak. Patients with mild symptoms have been advised for home treatment since most of the hospitals are running out of bed. In this situation delivering healthcare to people has become revolutionizing due to the rapid advancement of embedded systems, communication, and informatics technologies. Integration of different health sensors, handheld devices, and internet can be a great potential for significant improvement of the quality of remote healthcare. This paper discusses the use of MySignals HW shield which is a hardware development platform for medical devices to build e-health monitoring system. Wearable health monitoring system prototype has been developed in this work. To conduct experiments, health vitals such as body temperature, ECG, oxygen saturation level, and pulse rate from 5 volunteers have been measured, collected, and stored in a cloud database using the system prototype. To evaluate the performance of the prototype, transmission delay has been recorded in both wired (Ethernet) and wireless (Wi-Fi) communication modes. It is observed that it takes $2.71 \mathrm{~ms}$ and $5.18 \mathrm{~ms}$ of time to collect and store the health vitals to the cloud database in wired and wireless mode respectively. Comparing the collected health vitals with the normal range of health vitals, no abnormality is found in all volunteer's health. Finally, a framework for contactless monitoring of COVID-affected patients is proposed. Contactless monitoring of health vitals can reduce the chance of community spread.
\end{abstract}

Keywords Health monitoring · IoT · MySignals · Arduino · Wi-Fi · Wearable sensor · COVID 19

Suparna Biswas

mailtosuparna@gmail.com

Sohail Saif

sohailsaif7@gmail.com

Ramesh Saha

ramesh1saha@gmail.com

1 Maulana Abul Kalam Azad University of Technology, Kolkata, West Bengal, India

2 Gauhati University, Guwahati, Assam, India 


\section{Introduction}

E-health monitoring system can enhance the quality of living in elderly people with the help of advent wearable body sensors. According to a report by the World Health Organization, people more than 60 years of age will be increased to about 2 billion between 2015 and 2050 which is approx. $22 \%$ of the world population [1]. This alarming increase in the number of aged people will also increase chronic diseases, disability as well as financial burdens incurred due to hospitalization [2]. Chronic diseases like heart disease, stroke, cancer, diabetes are the main causes behind the deaths and these diseases should be treated early otherwise they can be life threatening. Recently world is witnessing the widespread of novel coronavirus (COVID-19) where millions of peoples are already affected due to this virus, thousands of peoples lost their life. People get easily infected by other COVID-19 patients in near proximity since it spreads through direct contact, coughs, sneeze droplets, etc. As a result of this outbreak, hospitals are experiencing a high volume of newly affected patients every day. In India, in a normal scenario, doctor to population ratio is 1:1800 [3] which is less when compared to the standard of 1:1000 [4] set by World Health Organization (WHO). Based on the current situation of COVID-19, doctors, nurses, and support staff could be affected while rendering treatment to the patients admitted in the hospitals suffering from this deadly virus. Here contactless monitoring of patients can reduce the chance of community spread.

Therefore considering the scenario of outbreak and increase of elderly people who often suffer from chronic age related ailments demanding continuous prolonged health monitoring, advanced technical solutions are required in the field of healthcare. Here, e-health monitoring can play an important role where traditional medical diagnosis can be integrated with new technologies, techniques and informatics [5-7]. Home hospitalization is one of the promising healthcare service which can be beneficial for the patients suffering from chronic diseases, by reducing the need to visit hospital repeatedly. This process has several benefits such as patients can enjoy continuous care in the family environment which can positively affect the benefit of medicine. Also it is economic, especially since the expense at the hospitals is increasing day by day, whereas home hospitalization decreases the expenses. In the process of Remote healthcare or home hospitalization, patient's must adhere to the doctor's advice as well patients health vitals need to be monitored periodically/continuously as needed [8].

Internet of Things (IoT) has the tremendous ability to connect real life objects to electronic devices to share information between them. This emerging technology has been widely accepted in several areas such as home automation, industry, transportation as well healthcare. It has been extensively applied in the process of remote health monitoring also its different technologies have greatly improved the quality of human life. Another emerging technology that is linked with IoT is cloud computing, this technology has delivered various resources such as ubiquitous computation, processing speed, storage, etc. With the help of various body sensors, health vitals can be collected and sent to the cloud platform for processing. Wearable sensors can gather the physiological parameters such as body temperature, heart rate, ECG, Oxygen level, etc. and send the data to the medical cloud using a smartphone as shown in Fig. 1. Cloud systems can perform several tasks such as data cleaning, data storage, data analysis. In the case of e-health monitoring systems doctors can see the patient data in real-time.

This paper designs and develops an e-health monitoring prototype using MySignals sensor kit test bed integrated with IoT and Cloud support. Also, architecture has been 


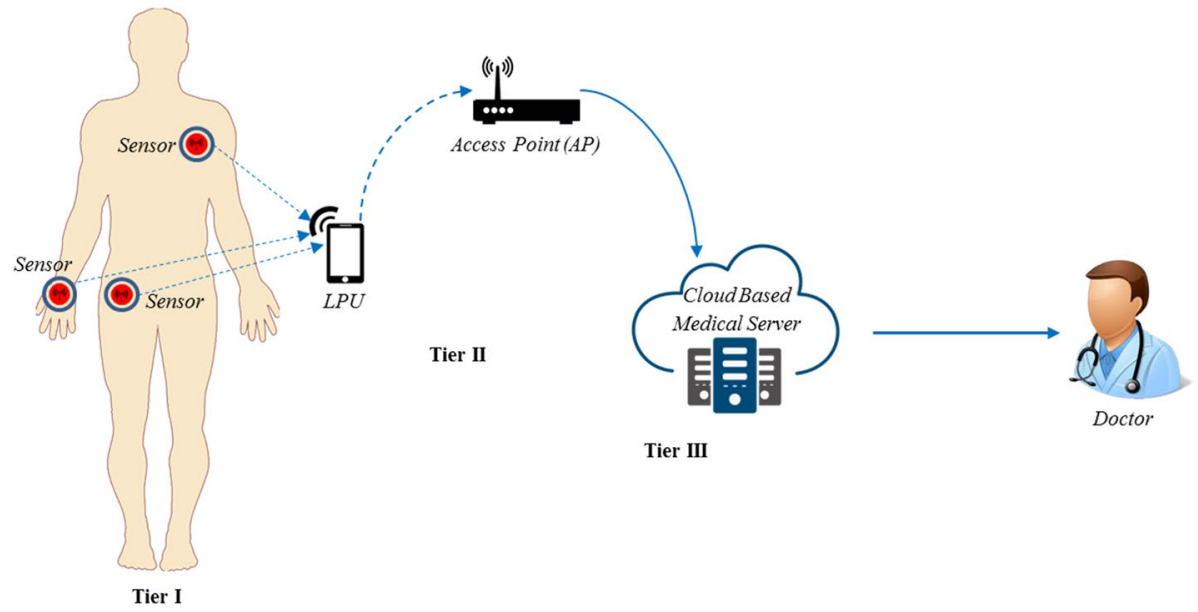

Fig. 1 Traditional architecture of body sensor based e-healthcare

proposed for contactless monitoring of patients suffering from COVID-19. The Rest of the paper has been organized as follows, Sect. 2 presents the components of MySignals HW shield including all health sensors. Recent related works on IoT-based health monitoring as well COVID patient monitoring have been presented in Sect. 3. Working prototype and its architecture have been described in Sect. 4 while Sect. 5 presents detailed analysis of experimental results. A framework for COVID -19 patient distant monitoring has been proposed in Sect. 6. Finally, Sect. 7 concludes the whole work.

\section{MySignals HW Development Platform}

Mysignals HW is a hardware development platform for medical device which is used to build e-health system. It is a package of 16 medical sensors which allows the user to measure several physiological parameters of the human body such as blood pressure, pulse, oxygen in the blood, breath rate, muscle electromyography signals, electrocardiogram signals, blood glucose levels, airflow, body temperature, body position, galvanic skin response, lung capacity, etc. Arduino UNO is integrated with MySignals HW as microcontroller which acts as a sink device as well gateway. Both wired and wireless communication is possible to connect the sensors with the device. Sensor data can be imported to a local server using serial communication also the data can be sent to a cloud medical server using Wi-Fi or GPRS module. Figure 2 shows the sensors and the type of connectivity available. Table 1 shows the technical specification of MySignals HW.

\subsection{Biomedical Sensors}

MySiganls HW contains several wearable medical sensors which are described below.

Blood pressure sensor This is a lightweight battery powered light weight sensor to measure the systolic and diastolic blood pressure in terms of millimeters of mercury 


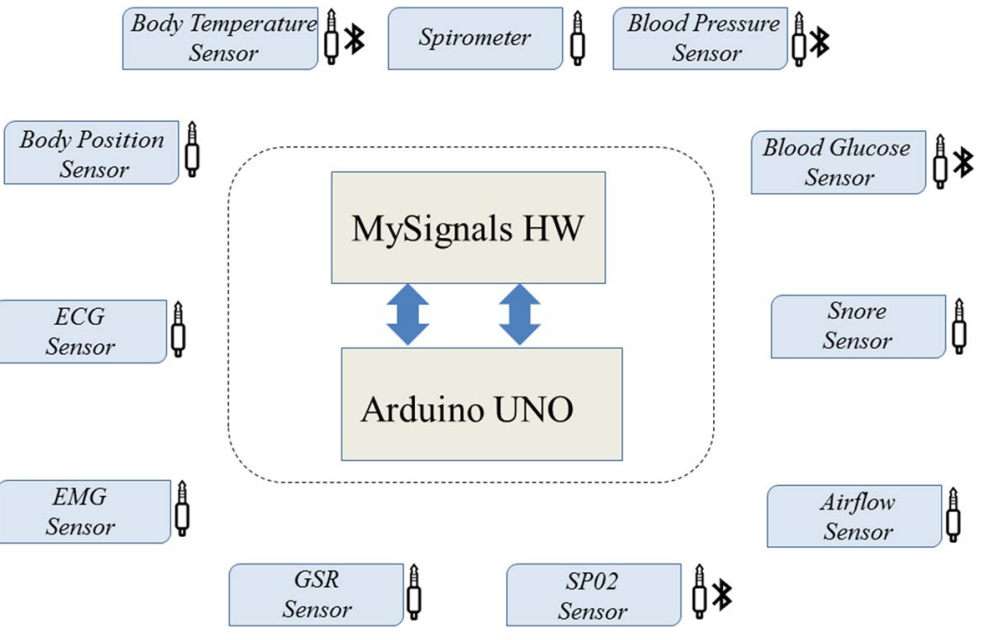

Fig. 2 MySignals HW and biomedical sensors

Table 1 Technical specifications of MySignals HW shield

\begin{tabular}{ll}
\hline Description & Values \\
\hline Processor & Atmega 328 (Arduino UNO) \\
RAM & $2 \mathrm{~K}$ \\
Flash memory & $32 \mathrm{~K}$ \\
UART sockets & 1 (multiplexed) \\
Radios on board & BLE, WiFi \\
Other supported radios & BT, ZigBee, 4G / 3G / GPRS \\
Communication mode & IP TCP/UDP \\
\hline
\end{tabular}

( $\mathrm{mmHg}$ ). Systolic blood pressure represents the pressure when the heart beats where diastolic pressure is the pressure on the blood vessels when the heart muscle is relaxed. Pulse oxygen (SPO2) sensor Pulse oximeter is used to measure the health of the cardiovascular and respiration system. It measures the oxygen saturation percentage in patient's blood as well as the heartbeats per minute.

Airflow breathing sensor This sensor is used to measure the rate of airflow from the nose/mouth which depicts the breathing rate. It is one of the important physiological parameters which helps the doctors to know the need for artificial respiration when a patient is in the critical situation.

Electromyography (EMG) sensor It is used to measure the muscle response or electrical activity in muscles when the cells in the muscle are activated electrically or neurologically. The signals are analyzed to detect certain neuromuscular abnormalities or to test the biomechanics of body movement.

Electrocardiogram (ECG) sensor It is used to record the electrical signals generated by the heart. Usually, three electrodes are placed over the chest in a different position to gather the electrical signals. This signal is analyzed to detect heart problems and monitor heart health. 
Blood glucose (Glucometer) sensor This battery-powered device is used to determine the approximate condition of the level of glucose in the blood. Strips containing a little amount of blood are used in the device. Usually, it is used to determine diabetes.

Spirometer air capacity sensor This is a portable small sensor device that is able to measure pulmonary functioning. It detects the volume of air inspired and expired by the lungs. Certain lung diseases such as asthma, bronchitis can be detected using the data gathered by the sensor.

Body temperature sensor This is a tiny wearable sensor attached to the human body that can detect body temperature with accuracy. Two metals are present in the sensor which generates electrical voltage or resistance once there is any change in temperature.

Body position (Accelerometer) sensor This sensor records the body position based on three axes. It is a wearable sensor attached to the body, thus it can detect the position such as stand, sit, up, down, left, right movement. This is very much beneficial for monitoring elderly peoples.

Galvanic skin response (GSR) sensor This sensor measures the electrical conductance of the skin based on the moisture level. Strong emotions can cause changes in sweat gland activity which varies the skin conductance. This is also a wearable sensor for the hands.

\section{Related Works}

In this section, the most significant and recent research works based on e-health monitoring and COVID patient monitoring which includes systems and solutions to monitor human health. Table 2 depicts a summary of the recent research works.

Hosseinzadeh et al. [9] proposed an IoT-based health monitoring system to monitor vital signals and identify the biological changes in elderly people. In the proposed IoT based approach, monitoring vital information of the subjects was the first task followed by an analysis of the collected data using various machine learning algorithms like decision tree (J48), Multilayer perceptron (MLP), Sequential Minimal Optimization (SMO) and Naïve Bayes (NB) classification for probability detection of physiological risk and behavioral changes in the subjects. It was concluded that J48 got maximum accuracy compared to the rest. Attaoui et al. [10] has proposed a wearable sensor based ECG telemedicine using Neural Networks combined with IoT. Here, human vital ECG signals are collected and measured using wearable sensor AD8232 which allows high frequency noise suppression. After collection, data is transmitted to the Gateway node (NRF24L01), where complex processing like baseline and linear variation is done. Then extraction of $\mathrm{R}$ peaks value using multilayer peak value is done, this $\mathrm{R}$ value determines the variation of the Heart rate. The Gateway and IoT platform together cooperate to provide virtual telemonitoring to the patients. Nayyar et al. [11] proposed a smart prototype of IoMT-based health monitoring system called as BioSenHealth 1.0. This prototype is used to collect health vitals of patients such as oxygen levels, pulse rate, heart rate, etc. in real-time and send live data to the doctor using IoT based web thingspeak.com. BioSenHealth have significantly improved with respect to the cost, accuracy, probability and fast response compared to the previous development prototype.

Leu et al. [12] proposed a real-time physiological health monitoring system using smartphone based wearable sensors. Here, physiological data is collected and transmitted to a smartphone in real-time, then the data is sent to the healthcare cloud using Wi-Fi. 


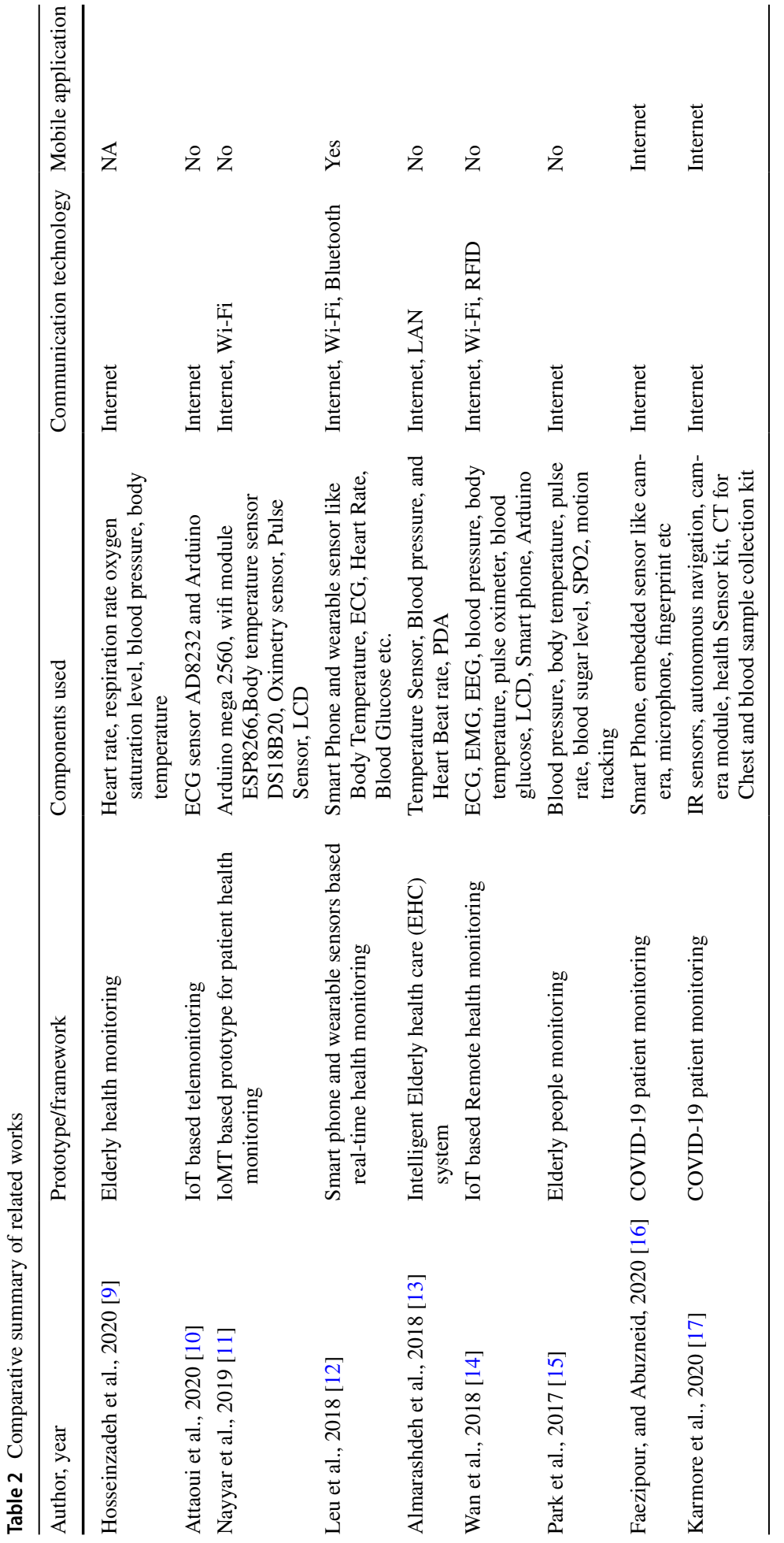


In the healthcare cloud the necessary classification and analysis is done. This individual information is then stored in the cloud. Authorized medical staff or doctors can access the data to monitor a patient's health condition and feedback is sent to the caregiver enabling them to reach the patient and provide the required assistance. Almarashdeh et. Al. [13] proposed an expert system for age old people for Elder Health Care (EHC). The main aim of this system is to reduce the high risk of elderly people who mainly suffer from specific chronic diseases. This system provides personalized care of the subjects' chronic diseases by using different wearable sensors such as Heart Rate, Body temperature and blood pressure. Authors proposed EHC architecture mainly three divisions or clients, first is elder people and the other two are nurse and physicians who take care of them. Wan et al. [14] developed a wearable IoT based real-time health monitoring system. Here, the author contributes mainly towards two objectives, first, a novel system: WISE (Wearable IoT-Cloudbased health system) which is used for real-time health monitoring. Here, several sensors are connected to the human body that collects vital information of the patient and analyzes them to give feedback to the caregiver. Secondly, the author has used a smartphone to process the data, visualize and transmit. In WISE, data is directly transferred to the medical cloud server; for a quick view of the real-time data author has included a lightweight wearable LCD for a quick view of real-time data.

Park et al. [15] proposed an IoT based Smart Healthcare Monitoring system for elderly adults. Here, wearable sensor devices are connected with each other to measure the vital parameters of the body, interpret the result and make an emergency alert to notify the doctor. Authors used specialized wearable sensors for developing elderly smart healthcare system like blood pressure, body temperature, pulse rate blood sugar level, SPO2, motion tracking, etc. They equipped wearable sensors in the elder people living room to monitor the emergency condition of the elder people or senior citizens. Sensors data are collected through mobile or PDA and send to the medical server through IoT based environment.

In [16] authors have proposed smart self-testing or monitoring for COVID-19 patients. With the use of smartphones, with embedded software, hardware, connectivity, and high computation capability many healthcare systems have been made possible. A careful observation of breathing sounds and its complication may indicate the COVID-19 virus and other similar viruses. In this application, the breathing sound is collected by the smartphone microphone and is stored in the repository. The stored data is analyzed using Machine/deep learning based pattern recognition techniques to separate the breathing phases, estimating the lung volume, oxygenation, and finally, classified as normal or abnormal. This smartphone based concept is to be used for self-monitoring of the ongoing world pandemic COVID-19 patients. Our proposed framework to monitor COVID affected patients using three wearable sensors to collect three important body vitals to classify normal and critical patients. Since three body vitals are considered to monitor the patients, the system can be more accurate instead of only using breathing sound analysis to detect the abnormalities in the patient health.

Karmore et al. [17] developed a Human Diagnosis Humanoid (MDH). It is an IoT-based software kit for the identification and diagnosis of COVID -19 suspects. This system prescribes a complete diagnostic test to the patient and verifies whether they are COVID-19 positive or not. The humanoid system uses the concept of real-time data sensing and processing through machine learning techniques with the help of different sensors. This system can identify the whereabouts of the patient; diagnose the individual as per COVID parameters and make a survey of the particular area of COVID patients.

Motivated by the literature, it can be observed that sensor based health monitoring can be very successful in managing patients suffering from COVID-19. With the use of 
a minimal number of biomedical sensors, it is possible to identify critical and normal patients in a hospital. In this paper, we have proposed an IoT based health monitoring framework designed especially for COVID affected patients where three biomedical sensor devices attached to the patient body can identify the severity of the disease and hence immediate action can be taken.

\section{Wearable e-health monitoring system prototype}

As a result of the above discussion, research and analysis, here we have presented a working prototype of e-health monitoring system. Three wearable biomedical sensors such as body temperature, SPO2 and ECG have been considered and MySignals HW has been used to build the health monitoring system. The Architecture of the proposed e-health monitoring prototype has been shown in Fig. 3. Here we have connected the sensor with MySignals HW using wired connection. Arduino UNO has been used with MySignals as a microcontroller. We have used both wired and wireless communication to transfer the health data to the cloud medical server and local server. To store the health data in the cloud server, MySQL database has been used. The cloud server used here is located in Mumbai, India and Amazon Web Services (AWS). Technical specification of the cloud server has been given in Table 3.

Body temperature sensors are placed on the left arm, Spo 2 sensor is placed on the right index finger and ECG probes are placed over the chest and stomach as shown in Fig. 4. These sensors are connected to MySignals HW using wire, $12 \mathrm{~V}$ power supply is given to Arduino UNO which is embedded with the MySignals HW. C++ based programming has been done and executed on Arduino which enables the sensors for data collection. For the experimental purpose, we have used both wired and wireless connection to forward the sensor data to the local or cloud server. In the case of wired connectivity Ethernet port has been used to connect the microcontroller with the network gateway. The network gateway or router is responsible for forwarding the data to the destination server. Initially, we have set an interval of $2 \mathrm{~ms}$ to collect the sensor data and store it in the local server.

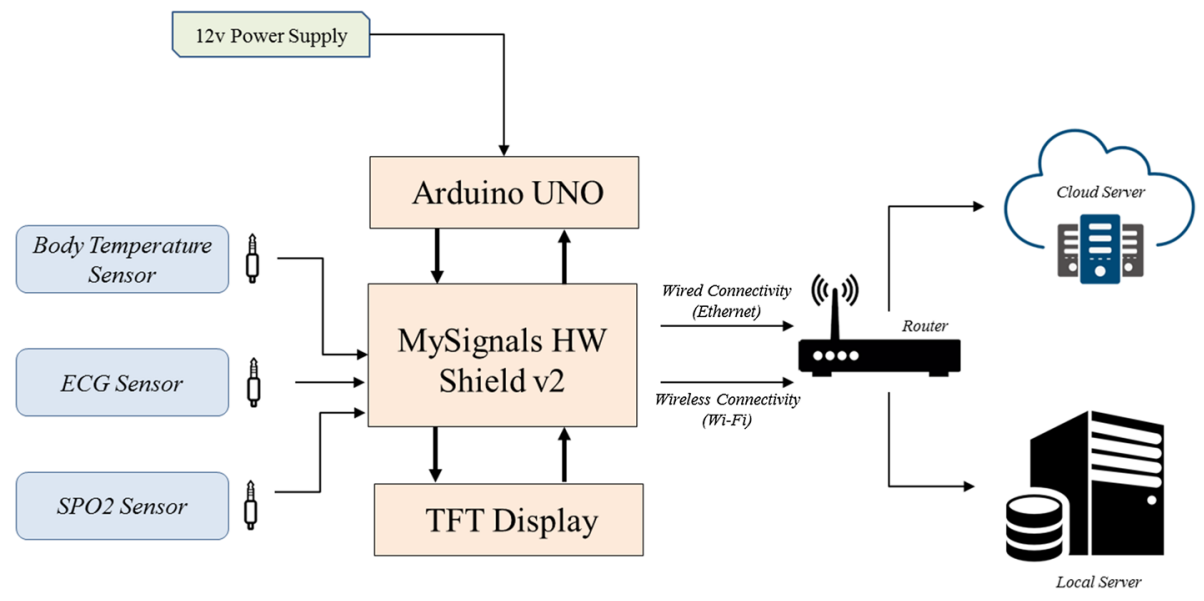

Fig. 3 Architecture of the proposed prototype 
Table 3 Technical specification of the cloud server

\begin{tabular}{ll}
\hline Description & Values \\
\hline Service & Amazon EC2 \\
No. of vCPU & 01 \\
Processor & Intel(R) Xeon(R) \\
& CPU E5-2676 v3 @ \\
& $2.40 \mathrm{GHz}$ \\
RAM & $1 \mathrm{~GB}$ \\
Storage & $30 \mathrm{~GB}$ (SSD) \\
OS & Ubuntu 18.04 \\
Web server & Apache 2.4.29 \\
Database server & MySQL \\
\hline
\end{tabular}

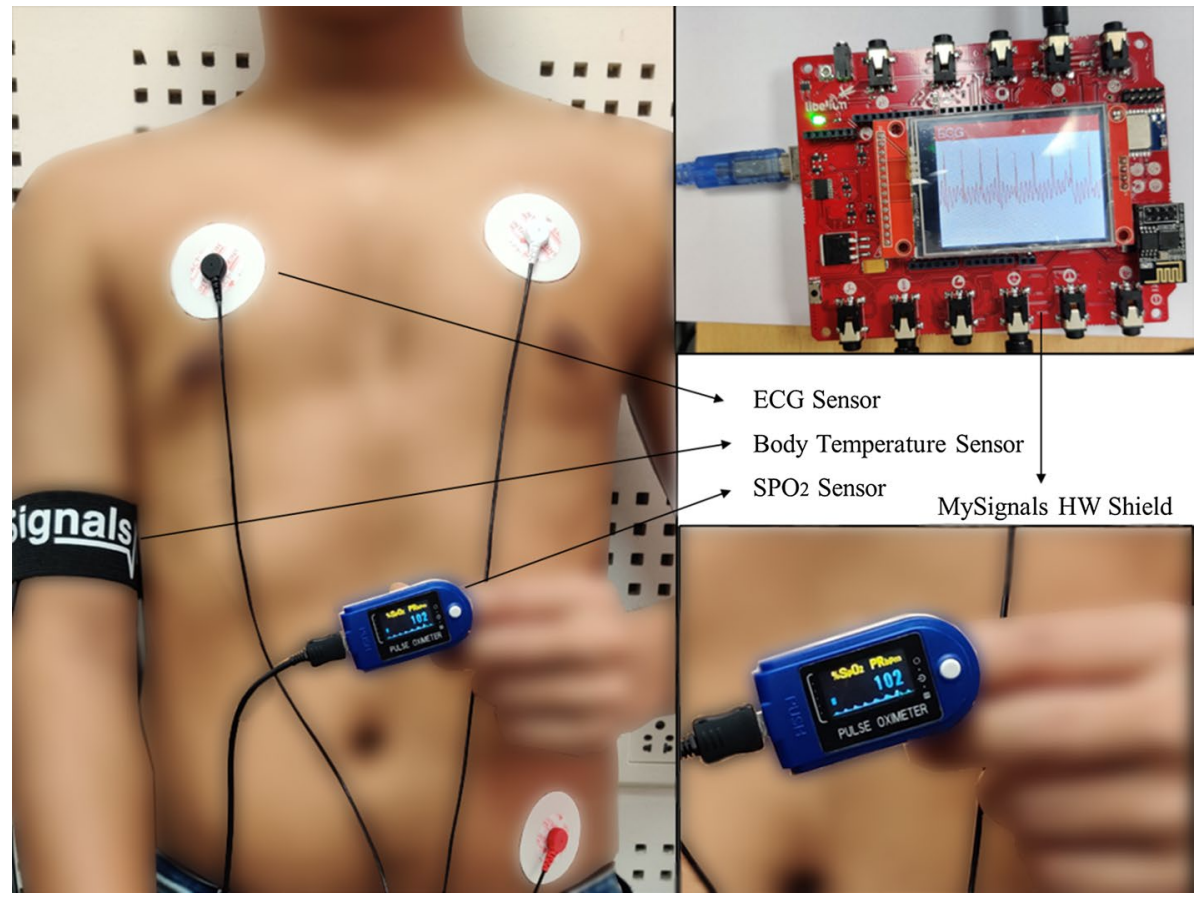

Fig. 4 Experimental setup for health vital collection

$\mathrm{SPO}_{2}$ sensor has a small display, which shows the oxygen saturation level and pulse rate, also the TFT display attached with the MySignals HW can show the data collected by sensors. We have also stored the health data in the cloud database. PHP based doctor's and patient's portal has been designed where both can login using their login credentials to view the health data. This will help the doctors to remotely monitor the patients and give necessary advice. 
Fig. 5 End-to-End delay for wired connection

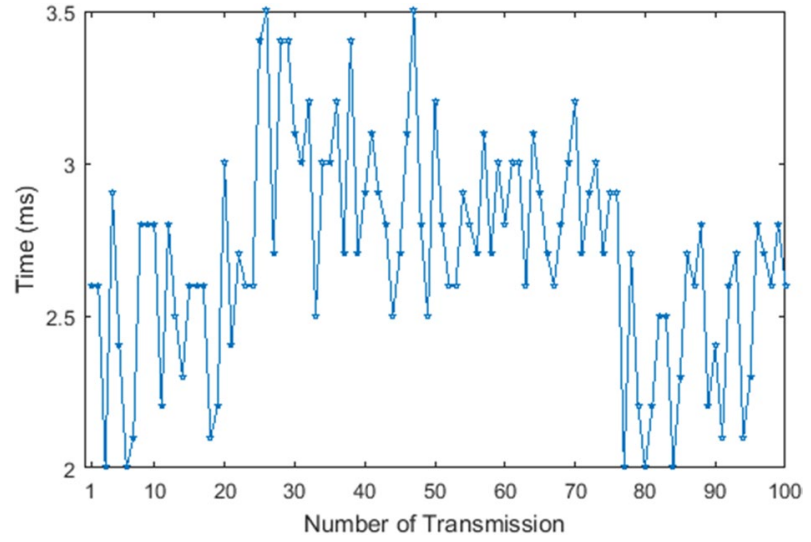

Fig. 6 End-to-end delay for wireless connection

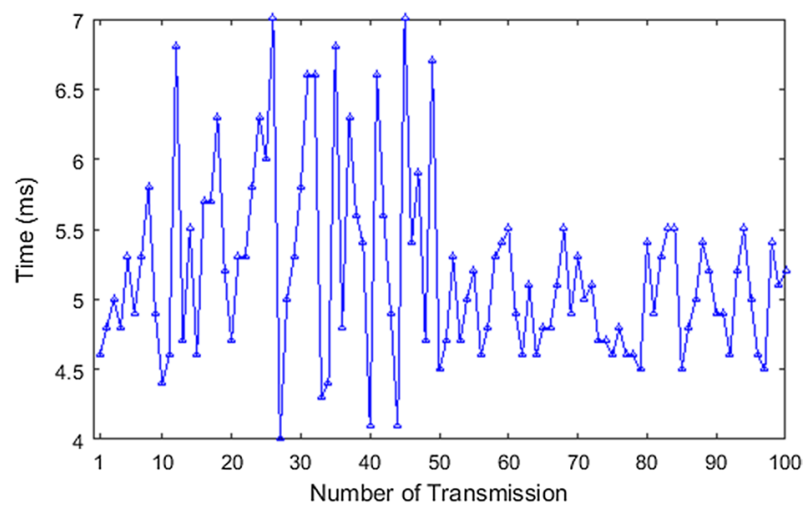

\section{Result and Analysis}

For our experiment, we have collected the health vitals of five healthy volunteers having normal health conditions using wearable sensors. Each record contains 5000 samples, hence a total of 25,000 samples have been collected. We have monitored each people for $60 \mathrm{~s}$ with an interval of $2 \mathrm{~ms}$. All the health vitals have been stored in the cloud database server. For the first $30 \mathrm{~s}$, we have collected the health vitals using wired connection and for the next $30 \mathrm{~s}$, Wi-Fi connection has been used to collect and store the data. We have recorded the average end-to-end transmission delay to collect the health vitals and store them in the cloud server for both wired and wireless connectivity. In Fig. 5 and 6 we have graphically plotted the transmission delay for 100 transmissions using wired and wireless connectivity respectively using Eq. 1.

$$
T_{d}=\frac{1}{N} \sum_{k=0}^{N}\left(R T_{k}-S T_{k}\right)
$$

where $N$ is the number of successful data transmission, $R T_{k}$ is the receiving time for packet $K$ and $S T_{k}$ is sending time for the same packet. 
Table 4 Comparison with other recent similar works

\begin{tabular}{lll}
\hline Author, Year & $\begin{array}{l}\text { No. of sensor } \\
\text { nodes }\end{array}$ & $\begin{array}{l}\text { End-to-end } \\
\text { delay (ms) }\end{array}$ \\
\hline Khan et al. [19], 2020 & 10 & 130 \\
Murtaza and Ali [20], 2020 & 80 & 70 \\
Ullah et al. [21], 2019 & 14 & 160 \\
Proposed & 03 & 2.71 \\
\hline
\end{tabular}

Table 5 Sample record set of the health data collected through the sensors

\begin{tabular}{llllll}
\hline & Elapsed time & Temperature & Pulse & SPO $_{2}$ & ECG \\
\hline Volunteer-1 & $0: 00.000$ & 99.2 & 72 & 95 & -0.03 \\
Age-28 & $0: 00.002$ & 99.2 & 72 & 96 & -0.035 \\
Gender-Male & $0: 00.004$ & 99.2 & 73 & 96 & -0.04 \\
Height-186 cm & $0: 00.006$ & 99.1 & 70 & 95 & -0.04 \\
Weight-72 kg & $0: 00.008$ & 99.2 & 72 & 99 & -0.035 \\
Volunteer-2 & $0: 00.000$ & 99.1 & 75 & 99 & -0.055 \\
Age-26 & $0: 00.002$ & 98.9 & 74 & 97 & -0.05 \\
Gender-Male & $0: 00.004$ & 98.9 & 75 & 97 & -0.05 \\
Height-179 cm & $0: 00.006$ & 98.5 & 74 & 98 & -0.05 \\
Weight-65 kg & $0: 00.008$ & 98.6 & 74 & 97 & -0.05 \\
Volunteer-3 & $0: 00.000$ & 98.1 & 75 & 96 & 0.0115 \\
Age-31 & $0: 00.002$ & 98.3 & 74 & 95 & 0.0115 \\
Gender-Male & $0: 00.004$ & 98.2 & 74 & 95 & 0.12 \\
Height-167 cm & $0: 00.006$ & 98 & 73 & 95 & 0.12 \\
Weight-62 kg & $0: 00.008$ & 98 & 72 & 96 & 0.125 \\
\hline
\end{tabular}

It can be observed from the plot that it takes an average of $2.71 \mathrm{~ms}$ of time for wired connectivity and $5.18 \mathrm{~ms}$ for Wi-Fi connectivity which is within the permissible limit of $125 \mathrm{~ms}$ for medical applications [18]. Table 4 presents the comparison of end-to-end delay with other similar works and it can be observed that our lab based prototype outperforms other similar works in terms of delay.

Four health parameters such as body temperature, pulse, oxygen saturation level and ECG have been considered during our experiments. Body temperature has been measured and recorded in Fahrenheit. For a typical adult normal range of body temperature is $97{ }^{\circ} \mathrm{F}$ to $99{ }^{\circ} \mathrm{F}$ whereas the range is $97.9^{\circ} \mathrm{F}$ to $100.4{ }^{\circ} \mathrm{F}$ for children [22]. Whenever the temperature crosses $100.4^{\circ} \mathrm{F}$, that condition is considered as fever. Body temperature may vary depending on conditions such as age, gender, food habit, activity etc. During our experiment, we have found all five volunteers are having normal body temperature. Table 5 shows a sample of a small record set of the health vitals collected for three volunteers.

ECG sensor is responsible to collect the ECG signals from the human body and then send those data to the storage server. Figure 7 shows the $10 \mathrm{~s}$ of ECG data plotted graphically for one of the volunteers. Traditional 12-lead systems are widely used to accurately capture ECG signals, However, too many electrodes may create discomfort for the patient.

According to a recent research article, a 3-lead placement in form of a triangle is enough to capture the primacy features of the ECG signal [23]. Typical ECG signals 


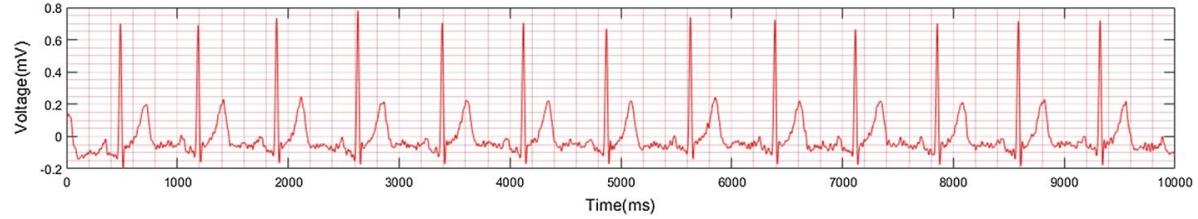

Fig. 7 ECG signal for $10 \mathrm{~s}$ time window

mainly contain five kinds of wave such as $\mathrm{P}, \mathrm{T}, \mathrm{Q}, \mathrm{R}$ and $\mathrm{S}$ wave. The interval between these waves such as RR,PR,QT interval and QRS complex are checked to diagnose various heart diseases. Normal values of the PR,RR,QT and QRS has been listed in Table 6 $[24,25]$.

Figure 8 shows a small portion of the ECG data, it can be observed that the intervals between the adjacent $\mathrm{R}$ waves are $0.71 \mathrm{~s}$ which depicts a healthy heart. QRS duration and QT interval are measured to be $0.08 \mathrm{~s}$ and $0.34 \mathrm{~s}$, which lies between the normal range also PR interval is within the normal range which indicates no abnormality in the heart. Based on the analysis of the ECG parameters, it can be deduced that no significant heart disease is present.

Table 6 Normal range of ECG features

\begin{tabular}{ll}
\hline Parameters & $\begin{array}{l}\text { Normal } \\
\text { values } \\
\text { (seconds) }\end{array}$ \\
\hline RR & $0.6-1$ \\
PR & $0.12-0.20$ \\
QT & $0.32-0.44$ \\
QRS & $<0.12$ \\
\hline
\end{tabular}

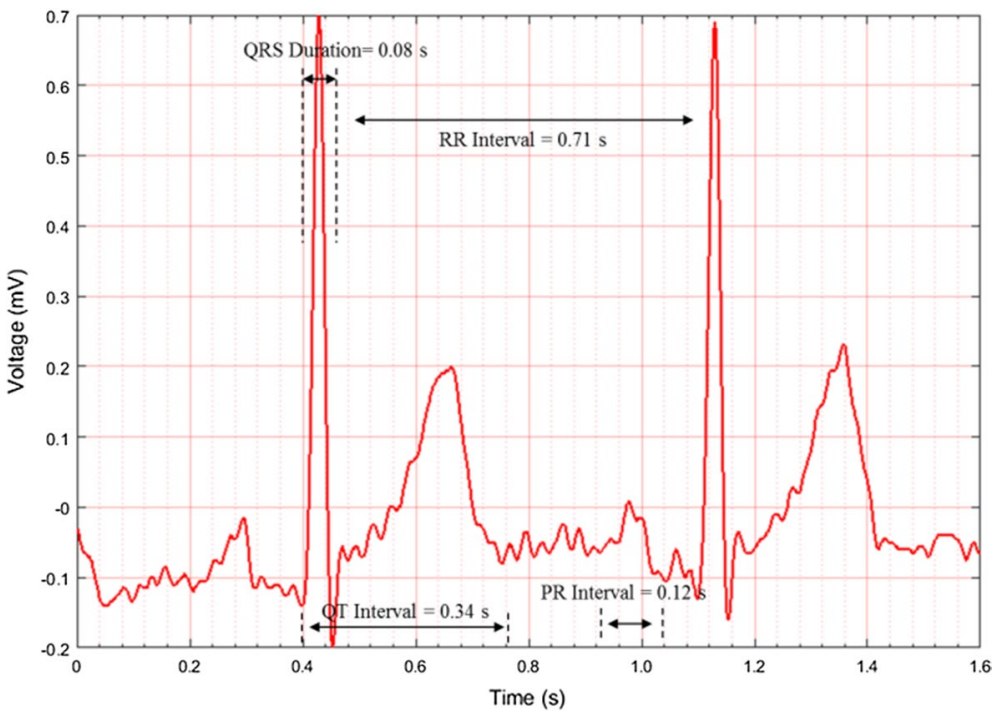

Fig. 8 Portion of the ECG signal 
Pulse rate and oxygen saturation level are other critical parameters for human health which motivated us to include those for our experiments. Here $\mathrm{SPO}_{2}$ sensor or Pulse Oximeter has been used to measure the pulse rate and oxygen percentage. During our experiment, we have also manually measured the pulse rates of our volunteers to verify the accuracy of the sensor device. Table 7 shows the comparison between the sensor measured pulse rate and manually measured pulse rate. Here we have found a significant difference between the manually measured and sensor measured values. To improve the accuracy of the sensor data, calibration is required and this is future scope of our work. The normal range of pulse rate for age group 6-12 years is $75-100$ whereas it is $60-100$ for more than 12 years [26]. Oxygen percentage in the blood is also measured using the same sensor and the data is recorded. Normal range for arterial blood oxygen saturation level is $95-100$, less than 90 percent can cause hypoxemia [27]. We have found that the oxygen saturation level for all of the five volunteer lies within 95-99 which depicts no abnormality.

\section{Proposed framework}

Here in this section, we have proposed a framework for contactless monitoring of patients suffering from COVID-19. Contactless monitoring can greatly reduce the chance of spread from patients to healthcare professionals. Sensor, IoT and cloud-based smart healthcare system can be effective to monitor patients' suffering from Corona Virus in the hospitals without any human intervention. IoT has an important role in different phases of this disease such as early diagnosis, quarantine time and after recovery [28]. Here we have proposed IoT based framework which can ensure contactless monitoring of patients as illustrated in Fig. 9. In the proposed framework we have used three biomedical sensors namely, $\mathrm{SpO}_{2}$, Body Temperature and Nasal Airflow Sensor, One Microcontroller device (Arduino), Mysignals Hardware shield and ESP8266 Wireless module for Arduino as illustrated in Fig. 9 and Fig. 10. According to World Health Organization (WHO) the symptoms of this virus affected disease are mainly Fever, Cough, Breathless-ness etc. So these sensors can collect health vitals such as Oxygen Saturation Level, Pulse Rate, Body Temperature and Respiratory rate. Those collected vitals will be sent to the cloud-based medical server through Wi-Fi and Internet. In the medical server, those data will be analyzed and alert will be sent to the respective caregiver's registered mobile number. As per WHO's guideline [29] a person with an acute corona infection, may include a history of fever or measured fever $\geq 38{ }^{\circ} \mathrm{C}, 100.4{ }^{\circ} \mathrm{F}$ ), cough, respiratory rate more than 30 breaths/ min and oxygen saturation $\left(\mathrm{SpO}_{2}\right)$ less than $90 \%$. Table 8 shows the values of body vitals depending on the condition of the patient.

So based on these health parameters and respective values provided by WHO we have considered threshold based mechanism to classify the patients in normal condition

Table 7 Manually measured and sensor measured pulse rate

\begin{tabular}{lllc}
\hline Volunteer & $\begin{array}{l}\text { Pulse rate (sen- } \\
\text { sor) }\end{array}$ & $\begin{array}{l}\text { Pulse rate } \\
\text { (manual) }\end{array}$ & Error (\%) \\
\hline Volunteer-1 & 74 & 68 & 8.10 \\
Volunteer-2 & 79 & 65 & 17.72 \\
Volunteer-3 & 71 & 68 & 4.22 \\
Volunteer-4 & 62 & 69 & 11.29 \\
Volunteer-5 & 64 & 69 & 7.81 \\
\hline
\end{tabular}




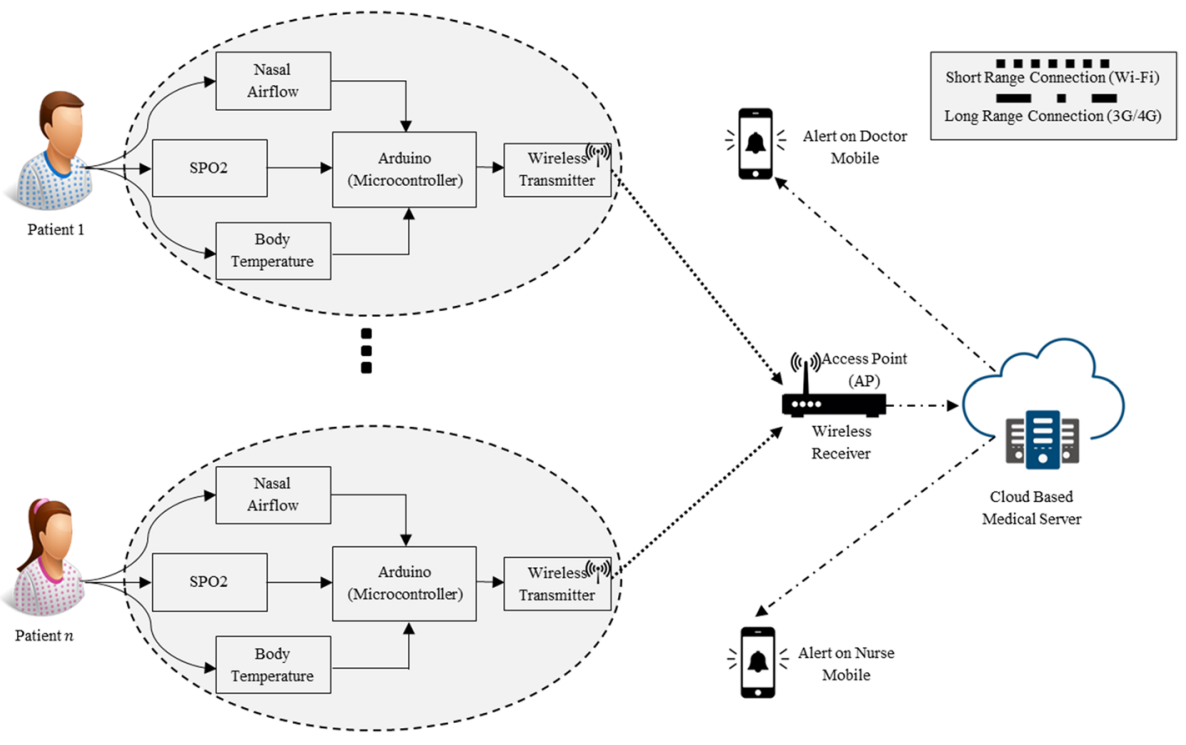

Fig. 9 Proposed framework for COVID patient monitoring

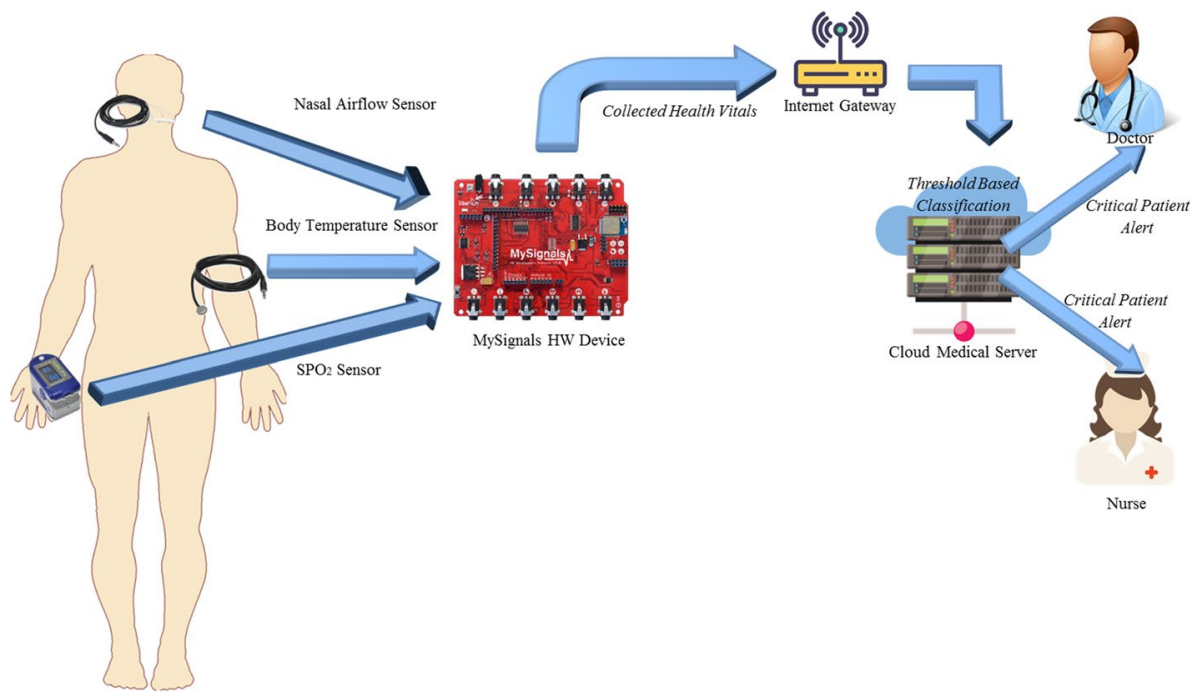

Fig. 10 MySignals HW device based framework for health vitals monitoring of COVID patients

Table 8 Clinical severity and assessment parameters

\begin{tabular}{llll}
\hline Condition & Oxygen saturation $\left(\mathrm{SPO}_{2}\right)$ & Body temperature $(\mathrm{BT})$ & Respiratory rate $(\mathrm{RR})$ \\
\hline Normal & $<94 \%($ range $90-94 \%)$ & $\geq 100.4{ }^{\circ} \mathrm{F}$ & $\geq 24 \mathrm{bpm}$ \\
Critical & $<90 \%$ & $\geq 101^{\circ} \mathrm{F}$ & $>30 \mathrm{bpm}$ \\
\hline
\end{tabular}


and critical patients who need immediate support as shown in Fig. 11. Our proposed framework can also be used in home quarantine or quarantine centers for continuous scanning of their health condition. Doctors, medical facilities and government agencies can also access the medical server to track patients.

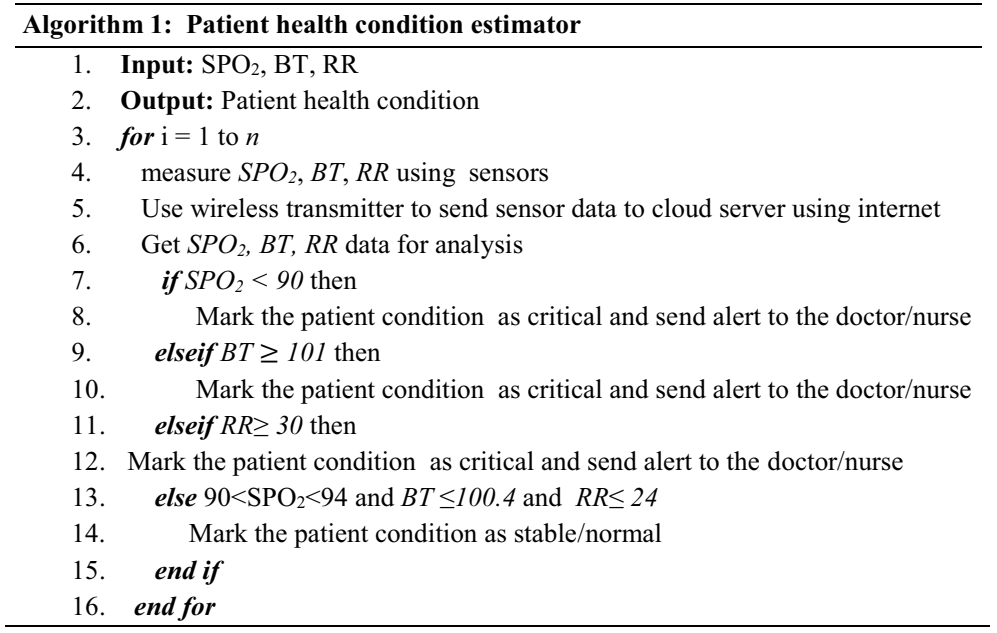

Here in the above framework we can see that wearable sensors have been used for the collection of physiological data. This system can work simultaneously for $n$ number of patients. Transmitter module associated with the microcontroller device is connected to the Internet access point (AP) through short-range communication such as Wi-Fi and that AP will forward the data to the medical server through $3 \mathrm{G}$ or $4 \mathrm{G}$ connectivity. In the medical server, data analysis is done and an alert is sent to the doctor's and nurse's mobile through $3 \mathrm{G}$ or $4 \mathrm{G}$ connectivity.

Fig. 11 Threshold based classification strategy

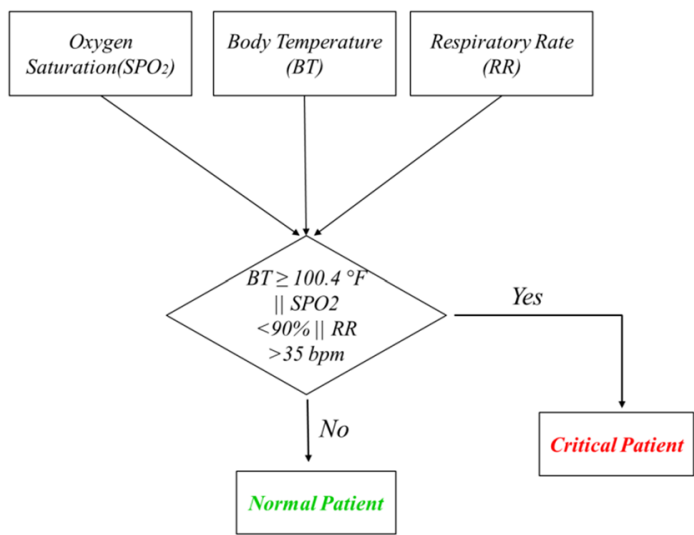




\section{Conclusion}

In this paper, we have discussed the use of the MySignals HW shield and various wearable biomedical sensors for remotely monitoring health vitals. A working prototype has been described where three sensors such as body temperature, ECG and SPO2 have been used to monitor health vitals and store the health data in local server as well cloud server. MySQL based cloud database has been used to store the health data, these data is accessible to patient and doctor. For experimental purpose wired and wireless connectivity has been used to send the health vitals from the MySignals device to the local/ cloud medical server. Health vitals of 5 healthy volunteers have been collected and stored using the prototype. To evaluate the performance of the prototype transmission delay has been recorded which depicts $2.71 \mathrm{~ms}$ and $5.18 \mathrm{~ms}$ of time is needed to collect and store the health vitals using wired and wireless connectivity respectively. Comparing the collected health vitals with the normal range of health vitals it is found that all health condition of all the volunteers is normal. This paper also proposes a framework utilizing the health monitoring prototype for contactless monitoring of patients suffering from COVID-19 to prevent the spread of this deadly disease among health professionals while exerting treatment and support. In the future, end-to-end system implementation along with sensor calibration and health data security can be targeted to achieve.

Funding This work has been carried out with grant received from WBDST sanctioned research project on secure remote healthcare with project sanction no. 230(Sanc)/ST/P/S\&T/6G-14/2018.

Availability of data and material Available but not shared due to privacy.

\section{Declarations}

Conflicts of interest Authors of this manuscript declare that they have no conflict of interest.

Code availability $\mathrm{C}++$ coding.

\section{References}

1. World Health Organization. (2015). World report on ageing and health. ISBN. 9789241565042.

2. García-Sánchez, P., González, J., Mora, A. M., \& Prieto, A. (2013). Deploying intelligent e-health services in a mobile gateway. Expert Systems with Applications, 40(4), 1231-1239.

3. Madhav, D. (2013). Doctor population ratio for India-The reality. The Indian Journal of Medical Research, 137, 632-635.

4. S. Anand, V. Fan "The Health Workforce in India" Human Health Resources for Health Observer Series No. 16 World Health Organization, Geneva, Switzerland, (2016).

5. Papa, A., Mital, M., Pisano, P., \& Giudice, M. D. (2020). E-health and wellbeing monitoring using smart healthcare devices: An empirical investigation. Technological Forecasting and Social Change, 153, 119226.

6. Jabirullah, M., Ranjan, R., Ali Baig, M. N., \& Kumar Vishwakarma, A. (2020). Development of e-health monitoring system for remote rural community of India. In 20207 th international conference on signal processing and integrated networks (SPIN), Noida, India (pp. 767-771).

7. Naranjo-Hernández, D., Reina-Tosina, J., \& Roa, L. M. (2020). Special issue "Body sensors networks for E-health applications." Sensors, 20, 3944. 
8. Hassen, H. B., Ayari, N., \& Hamdi, B. (2020). A home hospitalization system based on the Internet of things, Fog computing and cloud computing. Informatics in Medicine Unlocked, 20, 100368.

9. Hosseinzadeh, M., Koohpayehzadeh, J., Ghafour, M. Y., Ahmed, A. M., Asghari, P., Souri, A., Pourasghari, H., \& Rezapour, A. (2020). An elderly health monitoring system based on biological and behavioral indicators in internet of things. Journal of Ambient Intelligence and Humanized Computing. https://doi.org/10.1007/s12652-020-02579-7

10. El Attaoui, A., Hazmi, M., Jilbab, A., \& Bourouhou, A. (2020). Wearable wireless sensors network for ECG telemonitoring using neural network for features extraction. Wireless Personal Communications, 111(3), 1955-1976.

11. Nayyar, A., Puri, V., \& Nguyen, N. G. (2019). Biosenhealth 1.0: A novel internet of medical things (IOMT)-based patient health monitoring system. In International conference on innovative computing and communications (pp. 155-164). Springer, Singapore.

12. You, I., Choo, K. K. R., \& Ho, C. L. (2018). A smartphone-based wearable sensors for monitoring real-time physiological data. Computers \& Electrical Engineering, 65, 376-392.

13. Almarashdeh, I., Alsmadi, M., Hanafy, T., Albahussain, A., Badawi, U., Altuwaijri, N., Almaimoni, H., Asiry, F., Alowaid, S., Alshabanah, M., Alrajhi, D., Al Fraihet, A., \& Jaradat, G. (2018). Real-time elderly healthcare monitoring expert system using wireless sensor network. International Journal of Applied Engineering Research, 13, 3517-3523.

14. Wan, J., Al-awlaqi, M. A. A. H., Li, M., et al. (2018). Wearable IoT enabled real-time health monitoring system. Journal on Wireless Communications and Networking, 2018, 298.

15. Park, S. J., Subramaniyam, M., Kim, S. E., Hong, S., Lee, J. H., Jo, C. M., \& Seo, Y. (2017). Development of the elderly healthcare monitoring system with IoT. In Advances in human factors and ergonomics in healthcare (pp. 309-315). Springer.

16. Faezipour, M., \& Abuzneid, A. (2020). Smartphone-based self-testing of COVID-19 using breathing sounds. Telemedicine and e-Health, 26(10), 1202-1205.

17. Karmore, S., Bodhe, R., Al-Turjman, F., Kumar, R. L., \& Pillai, S. (2020). IoT based humanoid software for identification and diagnosis of Covid-19 suspects. IEEE Sensors Journal. https://doi. org/10.1109/JSEN.2020.3030905

18. Movassaghi, S., Abolhasan, M., Lipman, J., Smith, D., \& Jamalipour, A. (2014). Wireless body area networks: A survey. IEEE Communications Surveys \& Tutorials, 16(3), 1658-1686.

19. Khan, M. D., Ullah, Z., Ahmad, A., Hayat, B., Almogren, A., Kim, K. H., Ilyas, M., \& Ali, M. (2020). Energy harvested and cooperative enabled efficient routing protocol (EHCRP) for IoTWBAN. Sensors, 20, 6267.

20. Cicioglu, M., \& Çalhan, A. (2020). Energy-efficient and SDN-enabled routing algorithm for wireless body area networks. Computer Communications, 160, 228-239.

21. Ullah, Z., et al. (2019). Energy-efficient harvested-aware clustering and cooperative routing protocol for WBAN (E-HARP). IEEE Access, 7, 100036-100050.

22. Geneva, I. I., Cuzzo, B., Fazili, T., \& Javaid, W. (2019). Normal body temperature: A systematic review, open forum infectious diseases. Open Forum Infectious Diseases, 6(4), ofz032.

23. Wang, Y., et al. (2013). Design and evaluation of a novel wireless reconstructed 3-lead ECG monitoring system. In Proceedings IEEE 2013 biomedical circuits and systems conference (Bio-CAS) (pp. 362-365). Rotterdam.

24. Gertsch, M. (2009). The normal ECG and its (normal) variants. In The ECG manual (pp. 17-36). London: Springer.

25. ECGlibrary.com. (2016) Normal adult 12-lead ECG. In Ecglibrary.com. http://www.ecglibrary. com/norm.php.

26. Chirakanphaisarn, N., Thongkanluang, T., \& Chiwpreechar, Y. (2016). Heart rate measurement and electrical pulse signal analysis for subjects span of 20-80 years. Journal of Electrical Systems and Information Technology, 5, 112-120.

27. Macmillan, C., \& Andrews, P. (2000). Cerebrovenous oxygen saturation monitoring: Practical considerations and clinical relevance. Intensive Care Medicine, 26, 1028-1036.

28. World Health Organization. (2020). Clinical management of severe acute respiratory infection when COVID-19 is suspected. WHO/2019-nCoV/clinical/2020.4. March.

29. Nasajpour, M., Pouriyeh, S., Parizi, R. M., et al. (2020). Internet of things for current COVID19 and future pandemics: An exploratory study. Journal of Healthcare Informatics Research, 4, $325-364$.

Publisher's Note Springer Nature remains neutral with regard to jurisdictional claims in published maps and institutional affiliations. 

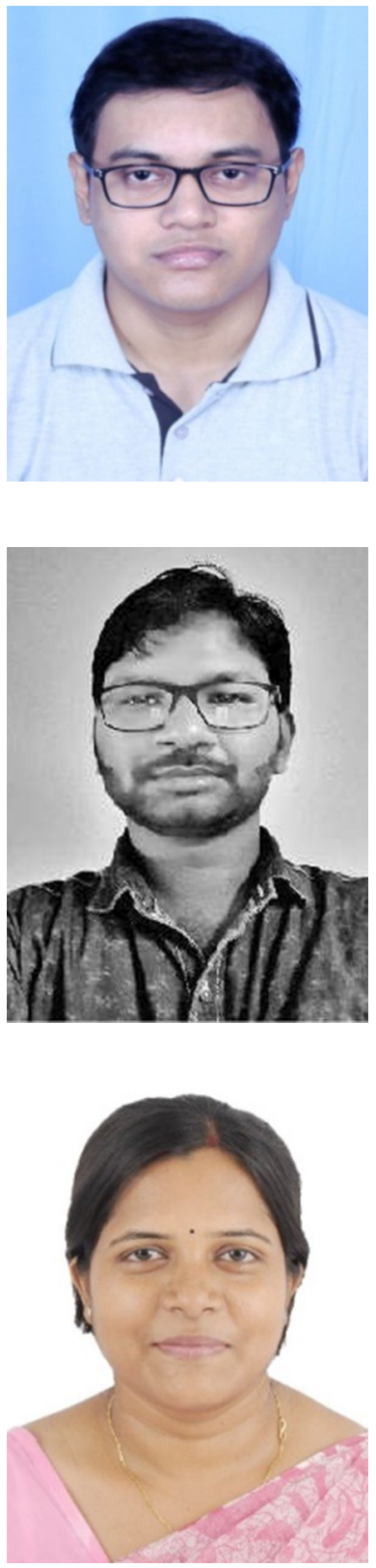

Sohail Saif is working as a Full Time Ph.D. Research Scholar at Maulana Abul Kalam Azad University of Technology, West Bengal, India. He completed his B.Tech. in Computer Science and Engineering and M.Tech. in Software Engineering from Maulana Abul Kalam Azad University of Technology, WB in 2014 and 2018, respectively. His areas of research interests are internet of things, network security and remote healthcare.

Mr. Ramesh Saha received the B.Tech. degree in Information Technology from Kalyani Govt. Engineering College, West Bengal, India in 2013. He received the M.Tech. degree from N.I.T.T.T.R., Kolkata, Kolkata, West Bengal, India in 2015. Currently, He is working as an Assistant Professor (Under TEQIP III) in the Department of Information Technology, GUIST, Gauhati University, Guwahati, India and pursuing Ph.D. from the Department of Computer Science and Engineering, Maulana Abul Kalam Azad University of Technology, West Bengal, India. He has authored a number of research papers in reputed journals and conferences of international repute. His areas of research interests IoT, IoV and e-heathcare. He is currently executing three funded research projects on IoT based healthcare system.

Dr. Suparna Biswas is an Associate Professor in the Department of Computer Science and Engineering in Maulana Abul Kalam Azad University of Technology, India. She completed her ME and Ph.D. from Jadavpur University, India in 2004 and 2013, respectively. She had been an ERASMUS MUNDUS Post Doctoral Research Fellow in cLINK project in Northumbria University, Newcastle, UK during 2014-2015. She has authored a number of research papers in reputed journals and conferences of international repute. Her areas of research interests include wireless networks, IoT and security. She is currently executing two funded research projects on IoT based remote healthcare in the capacity of principal and co-principal investigator. 